\title{
The Complete Flux Scheme for Spherically Symmetric Conservation Laws
}

\author{
J.H.M. ten Thije Boonkkamp and M.J.H. Anthonissen
}

\author{
Eindhoven University of Technology \\ Department of Mathematics and Computer Science \\ PO Box 513, 5600 MB Eindhoven, The Netherlands \\ \{j.h.m.tenthijeboonkkamp, m.j.h.anthonissen\}@tue.nl
}

\begin{abstract}
We apply the finite volume method to a spherically symmetric conservation law of advection-diffusion-reaction type. For the numerical flux we use the so-called complete flux scheme. In this scheme the flux is computed from a local boundary value problem for the complete equation, including the source term. As a result, the numerical flux is the superposition of a homogeneous flux and an inhomogeneous flux. The resulting scheme is second order accurate, uniformly in the Peclet numbers.
\end{abstract}

Keywords: finite volumes, advection diffusion equation, complete flux scheme.

\section{Introduction}

Many problems in physics and engineering can be modelled using conservation laws. These laws lead in general to a system of partial differential equations that cannot be solved analytically. Finite volume methods are a popular choice to discretise these equations, because they feature a discrete conservation property: the computational domain is divided into control volumes and on each volume a discrete conservation law holds.

In this paper we study a finite volume method for three-dimensional spherically symmetric steady conservation laws. This type of equation arises, e.g., in combustion theory, where the study of laminar spherical flames is useful for finding parameters such as burning velocity or flame curvature in premixed combustion [1].

Our model problem includes advection, diffusion and reaction terms and we shall develop a numerical scheme that is second order accurate for all flow conditions. This means that the scheme should always retain its high accuracy, unlike, e.g., standard exponentially fitted schemes, which are second order accurate for diffusion dominated flows but reduce to the first order upwind scheme when the advection term becomes large. Additionally the proposed scheme does not produce spurious oscillations for advection dominated flows, which is a well-known flaw of standard central discretisations.

High accuracy and the absence of wiggles are favourable properties that may also be achieved by using high resolution schemes such as flux limiting or 
(weighted) essentially nonoscillatory (ENO) methods [4. These techniques lead to larger discretisation stencils however which is disadvantageous. The method we present uses direct neighbours only.

Our algorithm is an extension of the finite volume methods for Cartesian grids introduced in 25,6] to spherically symmetric conservation laws. We use an exponential scheme for computing the numerical fluxes. The approximation for the flux is based on the complete differential equation. This implies that we also include the source term in the numerical fluxes.

Manzini and Russo [3] also present a finite volume method for advectiondominated problems that is second-order accurate away from boundary and internal layers. They pay special attention to the construction of the numerical advective fluxes in order to prevent numerical oscillations. This goal is achieved by a sophisticated reconstruction algorithm for cell gradients and a velocitybiased mixing of upwind and downwind contributions. Their scheme contains a nonlinear term for shock capturing.

This paper is organized as follows. In Section 2, we formulate a stationary advection-diffusion-reaction equation, introduce control volumes and formulate a second order discrete conservation law. In Section [3, we derive an expression for the numerical flux that is second order accurate for all flow conditions. In Section 4 we combine the discrete conservation law with the numerical flux and apply the resulting scheme to a spherically symmetric boundary value problem. We show numerical results using both the homogeneous and the complete flux scheme. By means of Richardson extrapolation we verify the order of accuracy of the finite volume scheme for different flow conditions.

\section{Finite Volume Discretization}

In this section we outline the finite volume method (FVM) for three-dimensional, spherically symmetric conservation laws.

Consider the following steady conservation law of advection-diffusion-reaction type, i.e.,

$$
\nabla \cdot(\boldsymbol{m} \varphi-\Gamma \nabla \varphi)=s
$$

where $\boldsymbol{m}$ is the mass flux, $\Gamma \geq \Gamma_{\min }>0$ a diffusion/conduction coefficient and $s$ a (chemical) source term. The unknown $\varphi$ can be, e.g., the temperature or the concentration of a species in a reacting mixture. The parameters $\Gamma$ and $s$ are usually (complicated) functions of the unknown $\varphi$, however, for the sake of discretisation we will consider these as given functions of the spatial coordinate $\boldsymbol{x}$. Equation (11) has to be coupled with the flow equations, i.e., the continuity equation and the momentum equations. The former reads

$$
\nabla \cdot \boldsymbol{m}=0
$$

Associated with (1), we introduce the flux vector $\boldsymbol{f}$, defined by

$$
\boldsymbol{f}:=\boldsymbol{m} \varphi-\Gamma \nabla \varphi
$$


Equation (11) then simply reduces to $\nabla \cdot \boldsymbol{f}=s$.

In a FVM we cover the domain with a finite number of control volumes $\Omega_{j}(j=$ $1,2, \ldots, N)$ and impose the integral form of the conservation law for each control volume, i.e.,

$$
\oint_{\partial \Omega_{j}} \boldsymbol{f} \cdot \boldsymbol{n} \mathrm{d} S=\int_{\Omega_{j}} s \mathrm{~d} V,
$$

where $\boldsymbol{n}$ is the outward unit normal on the boundary $\partial \Omega_{j}$. Next, we need numerical approximations for the integrals in (4). In the following, we assume the problem to be spherically symmetric, i.e., $\varphi=\varphi(r)$, and likewise for all other variables, and moreover, $\boldsymbol{f}=f(r) \boldsymbol{e}_{r}$ with $\boldsymbol{e}_{r}$ the first basis vector in spherical coordinates. We introduce a spatial grid $\left\{r_{j}\right\}$ of (uniform) grid size $\Delta r$. As control volumes we choose the spherical shells $\Omega_{j}:=\left(r_{j-1 / 2}, r_{j+1 / 2}\right)$ with $r_{j+1 / 2}:=\frac{1}{2}\left(r_{j}+r_{j+1}\right)$. Then, the surface integral in (4) reduces to

$$
\begin{aligned}
\oint_{\partial \Omega_{j}} \boldsymbol{f} \cdot \boldsymbol{n} \mathrm{d} S & =\int_{r=r_{j+1 / 2}} \boldsymbol{f} \cdot \boldsymbol{e}_{r} \mathrm{~d} S-\int_{r=r_{j-1 / 2}} \boldsymbol{f} \cdot \boldsymbol{e}_{r} \mathrm{~d} S \\
& =4 \pi\left(r_{j+1 / 2}^{2} f\left(r_{j+1 / 2}\right)-r_{j-1 / 2}^{2} f\left(r_{j-1 / 2}\right)\right) .
\end{aligned}
$$

For the approximation of the volume integral in (4) we apply the midpoint rule, to find

$$
\int_{\Omega_{j}} s \mathrm{~d} V \doteq \frac{4}{3} \pi\left(r_{j+1 / 2}^{3}-r_{j-1 / 2}^{3}\right) s_{j},
$$

with $s_{j}:=s\left(r_{j}\right)$. Combining (4), (15) and (6) and using the relation $x^{3}-y^{3}=$ $(x-y)\left(x^{2}+x y+y^{2}\right)$ we obtain the second order discrete conservation law

$$
r_{j+1 / 2}^{2} F_{j+1 / 2}-r_{j-1 / 2}^{2} F_{j-1 / 2}=\Delta r\left(r_{j}^{2}+\frac{1}{12} \Delta r^{2}\right) s_{j},
$$

where $F_{j+1 / 2}$ is the numerical flux at the cell interface approximating $f\left(r_{j+1 / 2}\right)$. Finally, the FVM has to be completed with the derivation of an expression for the numerical flux.

\section{Derivation of the Numerical Flux}

Our objective in this section is to derive an expression for the numerical flux that is uniformly second order accurate in the grid size, i.e., the discretisation error should always be second order for all flow regimes in combination with a source term of arbitrary strength.

We adopt the following notation: variables defined in the grid points $r_{j}$ and $r_{j+1}$ are indicated with the subscripts $\mathrm{C}$ and $\mathrm{E}$, respectively, and variables at the interface $r_{j+1 / 2}$ by the subscript e. The derivation of the expression for the numerical flux $F_{\mathrm{e}}$ at the eastern cell interface $r_{\mathrm{e}}$ located between the grid points $r_{\mathrm{C}}$ and $r_{\mathrm{E}}$ is based on the following model boundary value problem (BVP) for the unknown $\varphi$ :

$$
\begin{aligned}
& \frac{1}{r^{2}}\left(r^{2}\left(m \varphi-\Gamma \varphi^{\prime}\right)\right)^{\prime}=s, \quad r_{\mathrm{C}}<r<r_{\mathrm{E}}, \\
& \varphi\left(r_{\mathrm{C}}\right)=\varphi_{\mathrm{C}}, \quad \varphi\left(r_{\mathrm{E}}\right)=\varphi_{\mathrm{E}},
\end{aligned}
$$


where the prime $(')$ denotes differentiation with respect to $r$. In the derivation that follows, we assume that

$$
M:=r^{2} m=\text { Const }>0 \text { for } r \in\left(r_{\mathrm{C}}, r_{\mathrm{E}}\right) .
$$

Note that the condition $M=$ Const is a direct consequence of the continuity equation (2). The diffusion coefficient $\Gamma$ and the source term $s$ are arbitrary sufficiently smooth functions of $r$. The (scalar) flux corresponding to (8) reads

$$
f:=m \varphi-\Gamma \varphi^{\prime} .
$$

To derive the expression for $F_{\mathrm{e}}$ we carry out the following procedure:

1. Derive the integral expression for $\varphi(r)$ from the inhomogeneous BVP (8).

2. Derive the integral representation for $f\left(r_{\mathrm{e}}\right)$ from (10).

3. Approximate all integrals involved.

In the following, we need the variables $D, \lambda, \Lambda$ and $S$, defined by

$$
D(r):=\Gamma(r) r^{2}, \quad \lambda(r):=\frac{M}{D(r)}, \quad \Lambda(r):=\int_{r_{\mathrm{e}}}^{r} \lambda(\eta) \mathrm{d} \eta, \quad S(r):=\int_{r_{\mathrm{e}}}^{r} \eta^{2} s(\eta) \mathrm{d} \eta .
$$

The variable $\Lambda(r)$ is called the Peclet integral. Substituting (10) in (8aa) and integrating the resulting equation we obtain the following integral balance

$$
r^{2} f(r)-\left(r^{2} f\right)\left(r_{\mathrm{e}}\right)=S(r) .
$$

Using the definitions of $D$ and $\Lambda$ in (11), it is clear that the expression for the flux can be rewritten as

$$
r^{2} f(r)=-D\left(\varphi \mathrm{e}^{-\Lambda}\right)^{\prime} \mathrm{e}^{\Lambda} .
$$

Inserting this expression in (12) and once more integrating we obtain the following expression for the flux $f\left(r_{\mathrm{e}}\right)$ :

$$
\begin{aligned}
& \left(r^{2} f\right)\left(r_{\mathrm{e}}\right)=\left(r^{2} f^{(\mathrm{h})}\right)\left(r_{\mathrm{e}}\right)+\left(r^{2} f^{(\mathrm{i})}\right)\left(r_{\mathrm{e}}\right), \\
& \left(r^{2} f^{(\mathrm{h})}\right)\left(r_{\mathrm{e}}\right)=-\left(\mathrm{e}^{-\Lambda\left(r_{\mathrm{E}}\right)} \varphi_{\mathrm{E}}-\mathrm{e}^{-\Lambda\left(r_{\mathrm{C}}\right)} \varphi_{\mathrm{C}}\right) / \int_{r_{\mathrm{C}}}^{r_{\mathrm{E}}} D^{-1} \mathrm{e}^{-\Lambda} \mathrm{d} r, \\
& \left(r^{2} f^{(\mathrm{i})}\right)\left(r_{\mathrm{e}}\right)=-\int_{r_{\mathrm{C}}}^{r_{\mathrm{E}}} D^{-1} S \mathrm{e}^{-\Lambda} \mathrm{d} r / \int_{r_{\mathrm{C}}}^{r_{\mathrm{E}}} D^{-1} \mathrm{e}^{-\Lambda} \mathrm{d} r,
\end{aligned}
$$

where $\left(r^{2} f^{(\mathrm{h})}\right)\left(r_{\mathrm{e}}\right)$ and $\left(r^{2} f^{(\mathrm{i})}\right)\left(r_{\mathrm{e}}\right)$ are the homogeneous and inhomogeneous part, corresponding to the homogeneous and particular solution of (8), respectively.

We introduce some notation. $\langle a, b\rangle$ denotes the usual inner product of two functions $a(r)$ and $b(r)$ defined on $\left(r_{\mathrm{C}}, r_{\mathrm{E}}\right)$, i.e.,

$$
\langle a, b\rangle:=\int_{r_{\mathrm{C}}}^{r_{\mathrm{E}}} a(r) b(r) \mathrm{d} r .
$$




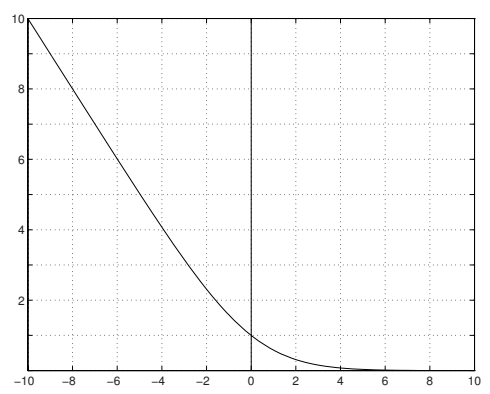

Fig. 1. The Bernoulli function $B(z)$

For a generic variable $v(r)$ defined on $\left(r_{\mathrm{C}}, r_{\mathrm{E}}\right)$ we indicate the geometric average (of $v_{\mathrm{C}}$ and $v_{\mathrm{E}}$ ) and the harmonic average by $\tilde{v}_{\mathrm{e}}$ and $\hat{v}_{\mathrm{e}}$, respectively, i.e.,

$$
\tilde{v}_{\mathrm{e}}:=\sqrt{v_{\mathrm{C}} v_{\mathrm{E}}}, \quad \frac{1}{\hat{v}_{\mathrm{e}}}:=\frac{\left\langle v^{-1}, 1\right\rangle}{\Delta r} .
$$

Consider the expression for the homogeneous flux. Assume first that $\Gamma(r)=$ Const on $\left(r_{\mathrm{C}}, r_{\mathrm{E}}\right)$. In this case expression (14b) reduces to

$$
\left(r^{2} f^{(\mathrm{h})}\right)\left(r_{\mathrm{e}}\right)=\frac{\tilde{D}_{\mathrm{e}}}{\Delta r}\left(B\left(-P_{\mathrm{e}}\right) \varphi_{\mathrm{C}}-B\left(P_{\mathrm{e}}\right) \varphi_{\mathrm{E}}\right)
$$

where $P_{\mathrm{e}}$ is the Peclet number defined by

$$
P_{\mathrm{e}}:=\frac{M \Delta r}{\tilde{D}_{\mathrm{e}}} .
$$

Furthermore, $B(z)$ is the Bernoulli function, defined by

$$
B(z):=\frac{z}{\mathrm{e}^{z}-1}
$$

see Figure 1. For the constant coefficient homogeneous flux, i.e., $\Gamma(r)$ and $M$ constant on $\left(r_{\mathrm{C}}, r_{\mathrm{E}}\right)$, we introduce the notation

$$
\left(r^{2} f^{(\mathrm{h})}\right)\left(r_{\mathrm{e}}\right)=\mathcal{F}^{\mathrm{h}}\left(\tilde{D}_{\mathrm{e}} / \Delta r, P_{\mathrm{e}} ; \varphi_{\mathrm{C}}, \varphi_{\mathrm{E}}\right)
$$

to denote the dependence of $\left(r^{2} f^{(\mathrm{h})}\right)\left(r_{\mathrm{e}}\right)$ on the parameters $\tilde{D}_{\mathrm{e}} / \Delta r$ and $P_{\mathrm{e}}$ and on the function values $\varphi_{\mathrm{C}}$ and $\varphi_{\mathrm{E}}$. In the general case, when $\Gamma(r)$ is an arbitrary function of $r$, we can rewrite the homogeneous flux in (14b) as

$$
\left(r^{2} f^{(\mathrm{h})}\right)\left(r_{\mathrm{e}}\right)=\mathcal{F}^{\mathrm{h}}\left(\hat{D}_{\mathrm{e}} / \Delta r,\langle\lambda, 1\rangle ; \varphi_{\mathrm{C}}, \varphi_{\mathrm{E}}\right) .
$$

Thus, the flux can be written as the constant coefficient flux with $\tilde{D}_{\text {e }}$ and $P_{\text {e }}$ replaced by $\hat{D}_{\mathrm{e}}$ and $\langle\lambda, 1\rangle$, respectively. 
Next, consider the inhomogeneous flux. Assume first that $\lambda(r)=$ Const on $\left(r_{\mathrm{C}}, r_{\mathrm{E}}\right)$ and define $P:=\lambda \Delta r$. Substituting the expression for $S(r)$ in (14c) and changing the order of integration, we find the following alternative representation for the inhomogeneous flux

$$
\left(r^{2} f^{(\mathrm{i})}\right)\left(r_{\mathrm{e}}\right)=\int_{r_{\mathrm{C}}}^{r_{\mathrm{E}}} G(\sigma(r) ; P) r^{2} s(r) \mathrm{d} r, \quad \sigma(r):=\frac{r-r_{\mathrm{C}}}{\Delta r},
$$

where $\sigma(r)$ is the normalized coordinate on $\left(r_{\mathrm{C}}, r_{\mathrm{E}}\right)$ and where $G(\sigma ; P)$ is the Green's function for the flux. It is given by

$$
G(\sigma ; P)=\left\{\begin{array}{cc}
\frac{1-\mathrm{e}^{-P \sigma}}{1-\mathrm{e}^{-P}} & \text { for } \quad 0 \leq \sigma \leq \frac{1}{2}, \\
-\frac{1-\mathrm{e}^{P(1-\sigma)}}{1-\mathrm{e}^{P}} & \text { for } \quad \frac{1}{2}<\sigma \leq 1 ;
\end{array}\right.
$$

see Figure2. Note that $G(\sigma ; P)$ relates the flux to the source term and is different from the usual Green's function, which relates the solution to the source term. If we furthermore assume that $s(r)=$ Const on $\left(r_{\mathrm{C}}, r_{\mathrm{E}}\right)$, relation (22) reduces to

$$
\left(r^{2} f^{(\mathrm{i})}\right)\left(r_{\mathrm{e}}\right)=\Delta r\left(\frac{1}{2}-W(P)\right) r_{\mathrm{C}}^{2} s+\mathcal{O}\left(\Delta r^{2}\right)
$$

where $W(z)$ is a weighting function, defined by

$$
W(z):=\frac{\mathrm{e}^{z}-1-z}{z\left(\mathrm{e}^{z}-1\right)}
$$

see Figure 3. From both figures, it is clear that the inhomogeneous flux is only of importance for advection dominated flow, i.e., $|P| \gg 1$, in combination with a large source term and in this case the upwind value $s_{\mathrm{C}}$ for $s(r)$ should be taken.

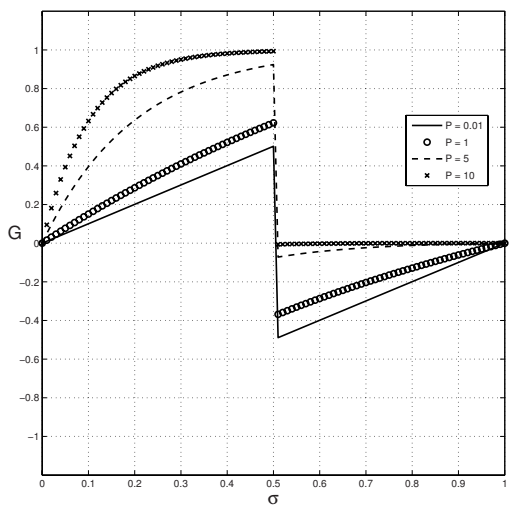

Fig. 2. Green's function $G(\sigma ; P)$ for several values $P>0$ 


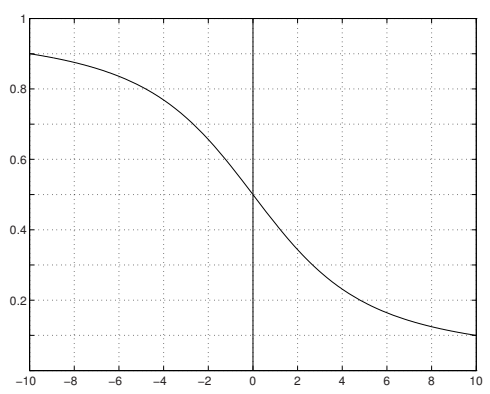

Fig. 3. The weighting function $W(z)$

For arbitrary functions $\Gamma(r)$ and $s(r)$ we have a similar representation for the inhomogeneous flux, i.e.,

$$
\left(r^{2} f^{(\mathrm{i})}\right)\left(r_{\mathrm{e}}\right)=\int_{r_{\mathrm{C}}}^{r_{\mathrm{E}}} G(\sigma(r) ;\langle\lambda, 1\rangle) r^{2} s(r) \mathrm{d} r,
$$

with $G(\sigma ; P)$ defined in (23) and where the normalized coordinate $\sigma(r)$ is defined by

$$
\sigma(r):=\int_{r_{\mathrm{C}}}^{r} \lambda(\eta) \mathrm{d} \eta /\langle\lambda, 1\rangle .
$$

Note that $\lambda(r)>0$ implying that $\sigma(r)$ is a monotonically increasing function on $\left(r_{\mathrm{C}}, r_{\mathrm{E}}\right)$. Expanding $s(r)$ in a Taylor series, we can also evalute the integral in (26a), to find

$$
\left(r^{2} f^{(\mathrm{i})}\right)\left(r_{\mathrm{e}}\right)=\Delta r\left(\frac{1}{2}-W(\langle\lambda, 1\rangle)\right) r_{\mathrm{C}}^{2} s_{\mathrm{C}}+\mathcal{O}\left(\Delta r^{2}\right) .
$$

Summarizing, we have the exact representation (21) for the homogeneous flux and the second order approximation (27) for the inhomogeneous flux. Both expressions hold for arbitrary $\Gamma(r)$ and $s(r)$. Since $\hat{D}_{\mathrm{e}} / \Delta r=M /\langle\lambda, 1\rangle$, the inner product $\langle\lambda, 1\rangle$ is the only integral that remains to be approximated. Straightforward integration and applying the mean value theorem of integration gives

$$
\langle\lambda, 1\rangle=\frac{M}{\Gamma\left(r^{*}\right)} \frac{\Delta r}{\tilde{r}_{\mathrm{e}}^{2}}, \quad r^{*} \in\left(r_{\mathrm{C}}, r_{\mathrm{E}}\right) .
$$

Using the approximation $\Gamma\left(r^{*}\right)=\tilde{\Gamma}_{\mathrm{e}}+\mathcal{O}(\Delta r)$ we obtain $\langle\lambda, 1\rangle=\tilde{P}_{\mathrm{e}}+\mathcal{O}\left(\Delta r^{2}\right)$. Inserting this approximations in the expressions (21) and (27) and omitting $\mathcal{O}\left(\Delta r^{2}\right)$-terms we obtain the following result for the numerical flux:

$$
\begin{aligned}
& \left(r^{2} F\right)_{\mathrm{e}}=\left(r^{2} F^{(\mathrm{h})}\right)_{\mathrm{e}}+\left(r^{2} F^{(\mathrm{i})}\right)_{\mathrm{e}}, \\
& \left(r^{2} F^{(\mathrm{h})}\right)_{\mathrm{e}}=\mathcal{F}^{\mathrm{h}}\left(\tilde{D}_{\mathrm{e}} / \Delta r, \tilde{P}_{\mathrm{e}} ; \varphi_{\mathrm{C}}, \varphi_{\mathrm{E}}\right), \\
& \left(r^{2} F^{(\mathrm{i})}\right)_{\mathrm{e}}=\Delta r\left(\frac{1}{2}-W\left(\tilde{P}_{\mathrm{e}}\right)\right) r_{\mathrm{C}}^{2} s_{\mathrm{C}},
\end{aligned}
$$

which is a second order approximation of (14). 


\section{Numerical Schemes and Example}

Combining the expressions (29) for the numerical flux with the discrete conservation law (7) we can derive two numerical schemes, i.e., the complete flux (CF) scheme and the homogeneous flux (HF) scheme, for which we only take into account the homogeneous part of the flux. We apply these schemes to a model BVP to investigate their performance for both diffusion dominated and advection dominated flow.

Substituting (29) in (7) we obtain the numerical scheme

$$
-a_{\mathrm{W}, j} \varphi_{j-1}+a_{\mathrm{C}, j} \varphi_{j}-a_{\mathrm{E}, j} \varphi_{j+1}=b_{\mathrm{W}, j} s_{j-1}+\left(b_{\mathrm{C}, j}+\Delta r\left(r_{j}^{2}+\frac{1}{12} \Delta r^{2}\right)\right) s_{j},
$$

with coefficients $a_{\mathrm{C}, j}$ etc., given by

$$
\begin{aligned}
& a_{\mathrm{W}, j}=\frac{\tilde{D}_{j-1 / 2}}{\Delta r} B\left(-P_{j-1 / 2}\right), \quad a_{\mathrm{E}, j}=\frac{\tilde{D}_{j+1 / 2}}{\Delta r} B\left(P_{j+1 / 2}\right), \quad a_{\mathrm{C}, j}=a_{\mathrm{W}, j}+a_{\mathrm{E}, j}, \\
& b_{\mathrm{W}, j}=\Delta r\left(\frac{1}{2}-W\left(P_{j-1 / 2}\right)\right) r_{j-1}^{2}, \quad b_{\mathrm{C}, j}=\Delta r\left(-\frac{1}{2}+W\left(P_{j+1 / 2}\right)\right) r_{j}^{2} .
\end{aligned}
$$

For the HF scheme we have to take $b_{\mathrm{W}, j}=b_{\mathrm{C}, j}=0$. Note that both schemes gives rise to a tridiagonal system $\mathbf{A} \varphi=\mathbf{B s}$, which can be very efficiently solved using LU-decomposition.

Consider the following BVP

$$
\begin{aligned}
& \frac{1}{r^{2}} \frac{\mathrm{d}}{\mathrm{d} r}\left(M \varphi-\Gamma r^{2} \frac{\mathrm{d} \varphi}{\mathrm{d} r}\right)=s, \quad 0<r<1, \\
& \varphi(0)=5, \quad \frac{\mathrm{d} \varphi}{\mathrm{d} r}(1)=0,
\end{aligned}
$$

with $\Gamma(r)$ and $s(r)$ given by

$$
\Gamma(r)=\Gamma_{\min }(1+\sqrt{r}), \quad s(r)=\frac{s_{\max }}{1+s_{\max }(2 r-1)^{2}} .
$$

The diffusion coefficient $\Gamma(r)$ is a smoothly varying function whereas the source term has a sharp peak, introducing a steep interior layer near $r=\frac{1}{2}$; see Figure 4

To assess the order of accuracy of both schemes, we compute numerical approximations of $\varphi\left(\frac{1}{2}\right)$ with increasingly smaller grid sizes and apply Richardson extrapolation to these results. More precisely, let

$$
\varphi\left(\frac{1}{2}\right)=\varphi^{h}+\varepsilon^{h}=\varphi^{h / 2}+\varepsilon^{h / 2}=\varphi^{h / 4}+\varepsilon^{h / 4}, \quad h=\Delta r,
$$

where $\varphi^{h}$ denotes the numerical approximation of $\varphi\left(\frac{1}{2}\right)$ computed with grid size $h$ and $\varepsilon^{h}$ the corresponding (global) discretisation error, etc. Assuming the following expansion

$$
\varepsilon^{h}=C h^{p}+\mathcal{O}\left(h^{q}\right), \quad q>p,
$$

we can derive the following expression for the order of accuracy $p$ :

$$
2^{p} \doteq \frac{\varphi^{h / 2}-\varphi^{h}}{\varphi^{h / 4}-\varphi^{h / 2}}=: q^{h}
$$




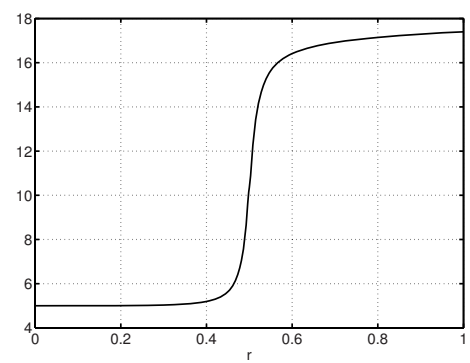

Fig. 4. Solution of the model BVP (31). Parameter values are: $M=1, \Gamma_{\min }=10^{-7}$ and $s_{\max }=10^{3}$.

Table 1. The $q^{h}$-values for the complete flux scheme and the homogeneous flux scheme as a function of $N=1 / h$. Parameter values are: $M=1$ and $s_{\max }=10^{3}$.

\begin{tabular}{|c|c|c|c|c|}
\hline & \multicolumn{2}{|c|}{$\Gamma_{\min }=10^{-1}$} & \multicolumn{2}{c|}{$\Gamma_{\min }=10^{-7}$} \\
\hline$N$ & HF & CF & HF & CF \\
\hline \hline 10 & 2.82 & 2.57 & 2.37 & 2.99 \\
20 & 5.56 & 5.65 & 2.70 & 6.59 \\
40 & 10.03 & 12.67 & 2.31 & 18.08 \\
80 & 4.92 & 5.24 & 2.03 & 6.07 \\
160 & 4.07 & 3.97 & 2.01 & 4.07 \\
320 & 4.02 & 3.96 & 2.00 & 4.02 \\
640 & 4.01 & 3.98 & 2.00 & 4.00 \\
1280 & 4.02 & 4.01 & 2.00 & 4.00 \\
\hline
\end{tabular}

In Table 1 you find the values of $q^{h}$ for both schemes. Clearly, when diffusion is dominant, i.e., for $\Gamma_{\min }=10^{-1}$, both schemes behave second order accurate. Thus both schemes perform equally well, in agreement with the previous observation that the inhomogeneous flux is only of importance for advection dominated flow. On the other hand, for dominant advection, i.e., $\Gamma_{\min }=10^{-7}$ the homogeneous flux scheme reduces to first order, whereas the complete flux scheme is still second order.

\section{Conclusions and Future Research}

In this paper we have derived the complete flux scheme for spherically symmetric conservation laws of advection-diffusion-reaction type. The numerical flux is computed from a local BVP for the entire equation, including the source term. All parameters are assumed to be arbitrary, sufficiently smooth functions of the radial coordinate $r$. As a result, the numerical flux is the superposition of a homogeneous flux, corresponding to the homogeneous solution of the BVP, and an inhomogeneous flux, corresponding to the particular solution. The resulting scheme behaves second order accurate, uniformly in the Peclet number, does not 
introduces numerical oscillations near a steep layer and has a simple three-point stencil.

Directions for further research are the following. A first obvious extension is to apply the scheme to time dependent conservation laws. An option would be to include the time derivative in the inhomogeneous flux and subsequently apply a suitable time integration method. A second extension is to apply the scheme to the conservation laws of a spherically symmetric flame. Since our model BVP has an interior layer reminiscent of a flame front, it is expected that the CFscheme will give accurate results for laminar flames. The major problem in this case is to construct fast and robust iterative methods to solve the nonlinear, discrete system. A final extension the authors have in mind is to simulate time dependent, i.e., expanding or imploding, spherical flames. All these issues will be subject of future research.

\section{References}

1. Groot, G.R.A.: Modelling of Propagating Spherical and Cylindrical Premixed Flames. PhD Thesis, Eindhoven University of Technology (2003)

2. Van 't Hof, B., Ten Thije Boonkkamp, J.H.M., Mattheij, R.M.M.: Discretisation of the stationary convection-diffusion-reaction equation. Numer. Meth. for Part. Diff. Eq. 14, 607-625 (1998)

3. Manzini, G., Russo, A.: A finite volume method for advection-diffusion problems in convection-dominated regimes. Comput. Methods Appl. Mech. Engrg. 197, 12421261 (2008)

4. Shu, C.-W.: High-order finite difference and finite volume WENO schemes and discontinuous Galerkin methods for CFD. International Journal of Computational Fluid Dynamics 17(2), 107-118 (2003)

5. Thiart, G.D.: Finite difference scheme for the numerical solution of fluid flow and heat transfer problems on nonstaggered grids. Numerical Heat Transfer, Part B 17, 43-62 (1990)

6. Thiart, G.D.: Improved finite difference scheme for the solution of convectiondiffusion problems with the SIMPLEN algorithm. Numerical Heat Transfer, Part B 18, 81-95 (1990) 